\title{
EVOLUTIONARY MECHANICS OF PROTRUSIBLE TENTACLES AND TONGUES
}

\author{
by
}

\author{
JOHAN L. VAN LEEUWEN ${ }^{1, *}$, JURRIAAN H. DE GROOT $^{2}$ \\ and WILLIAM M. KIER ${ }^{3}$
}

\section{$\left({ }^{1}\right.$ Experimental Zoology Group, Wageningen Institute of Animal Sciences, Wageningen University, Marijke 40, NL-6709 PG Wageningen, The Netherlands $;{ }^{2}$ Institute for Evolutionary and Ecological Sciences, Leiden University, The Netherlands; ${ }^{3}$ Department of Biology, University of North Carolina, Chapel Hill, USA)}

\section{ABSTRACT}

This paper provides a comparison at multiple levels of structural organization of the biomechanics of protrusible muscular systems with different origins and phylogenetic history. The high-performance prey capture tentacles in squid, the tongues of frogs and salamanders, and the tongue of the chameleon are used as examples.

The tentacles of squid are muscular organs that lack bony elements. They are rapidly elongated during prey capture (typical extension time $25 \mathrm{~ms}$, peak acceleration of approximately $250 \mathrm{~m} . \mathrm{s}^{-2}$ ) by extensor muscles that have remarkably short sarcomeres (myosin filaments are only 0.5 to $1.0 \mu \mathrm{m}$, compared with $1.6 \mu \mathrm{m}$ in vertebrates). Short sarcomeres generate only relatively small forces, but relatively high absolute strain rates for a given interfilamentary sliding velocity (at low external loads). A forward dynamics model (VAN LEEUWEN \& KIER, 1997) predicts the movements of the tentacles with reasonable accuracy and predicts also that the short sarcomeres provide optimal extension velocity.

Several frogs (Hemisotidae and Microhylidae) have a similar extension mechanism in their tongue (denoted as hydrostatic elongators by NISHIKAWA, 1999b) to that found in the tentacles of squid. The extension performance is, however, limited relative to that observed in squid. This can be explained by two factors. First, the extensor fibres run in one direction only, while in the squid the fibres are arranged in circumferential arcs as well as in two other fibre groups that run at right angles to each other. The unidirectional fibre orientation results in a smaller extension for a given shortening of the extensor fibres than observed in the tentacle. Second, the myosin filaments in the extensor muscle of the frog tongue are similar to those found in other vertebrate skeletal muscle. It is likely that the filament lengths are not optimised for a high peak extension velocity although data are lacking thus far. These limitations have been circumvented by several groups of frog (Bufonidae, Ranidae and others) that 'throw' their tongue out of the mouth by a rapid jaw movement (inertial elongators).

The ballistic tongues of many plethodontid salamanders extend in less than $10 \mathrm{~ms}$. The most extreme performance is found in Hydromantes supramontis, which elongates the tongue up to $80 \%$ of body length. The paired cylindrical protractor muscles envelope two elongate epibranchials, the posterior 'legs' of the tongue skeleton. The complete tongue skeleton is projected with the tongue pad. The normal stress of the protractor muscle on

* To whom correspondence should be addressed: email: johan.vanleeuwen@morf.edc. wag-ur.nl 
the tapered epibranchials is postulated to be the main projection force (DEBAN et al., 1997). We suggest that a build up of a 'pre-stress' in the protractor and an associated storage of elastic energy in connective tissues prior to projection may be essential for rapid projection. Detailed kinematic studies of plethodontid tongue projection are needed.

The ballistic chameleon tongue has a remarkable performance, with a reported peak acceleration of the tongue during prey capture of about $500 \mathrm{~m} \cdot \mathrm{s}^{-2}$. A cylindrical accelerator muscle envelopes the elongated entoglossal bone and is projected out of the mouth with the tongue pad. The extreme performance is likely due to a combination of several factors. First, the arrangement of muscle fibres in spiral arcs allows for close packing and uniform work output of the accelerator muscle fibres (VAN LEEUWEN, 1997). Second, pre-stress in hyobranchial muscles and elastic energy storage in connective tissues prior to projection may also be an essential element.

In future work, more attention should be paid to the possibility of elastic energy storage mechanisms in high-performance protrusible tentacles and tongues.

KEY WORDS: chameleon, frog, salamanders, squid, toad, dynamics, kinematics, tentacles, tongues.

\section{INTRODUCTION}

This paper reviews mechanical aspects of protrusible muscular organs that are used to capture prey: tentacles of squid and the tongues of amphibians and reptiles. These organs typically consist of tightly packed, three-dimensional arrays of muscle fibres and they are often capable of a very rapid change in shape, lasting for instance only $10-100 \mathrm{~ms}$ and leading to longitudinal muscle fibre strains of up to 0.8. The biomechanics of these muscular systems is complex. Activation of the muscle fibres may cause large deformations, which are generally difficult to predict because of the fibre-fluid nature of the tissues and the non-linear properties of these components. The high number of degrees of freedom of movement of these systems leads potentially to complex and distributed control.

Several important questions arise in the study of protrusible muscular systems. Examples are:

1. The performance of a protrusible muscular system depends heavily on the control and integrated design of several tissues at many structural levels (molecule, organelle, cell, tissue, organ). How are the different architectural levels integrated to yield a functional system?

2. How can the extension dynamics be understood in the light of muscle contraction dynamics ( strain rate, power output etc.), intramuscular pressure development, and elastic energy storage and release mechanisms. 
3. What is the effect of the external medium (tentacles are extended in water, the tongue of the chameleon is projected in air) on the performance and design?

4. What are the phylogenetic constraints for the various constructions and their components?

The design and performance of tentacles and tongues will be discussed in the light of the above questions and a conceptual frame work will be presented in the next section.

\section{CONCEPTUAL FRAME WORK}

To understand the architecture and function of protrusible muscular organs a number of steps must be taken. The following steps can be addressed as an example:

1. Measure architecture and tissue properties. This includes anatomical measurements at various levels (from the molecular to macroscopical level), physiological measurements of the properties of muscle fibres, measurements of the activation distribution in the muscles, etc.

2. Make quantitative models that predict aspects of the design of the system (such as the optimal arrangement of the muscle fibres) or the deformation of the muscular system (with a forward dynamics model) using information obtained in step 1. Prediction of optimized architectures must be handled carefully, because optimality may be limited by phylogenetic constraints.

3. Perform experimental tests. This might include for instance anatomical observations of the muscle fibre arrangement, measurement of intramuscular pressure or measurement of the deformation and extension speed of the muscular system.

4. Investigate phylogenetic aspects with cladistic methods (using for instance molecular methods, more traditional anatomical methods or combinations ) and compare the mechanical design and performance between different clades (see for instance NisHIKAWA, 1999b). Similar selection pressures are expected to lead frequently to similar solutions.

From the above list, it is clear that an interdisciplinary approach is required.

To understand the complexities of muscular protrusible systems, the development of quantitative models is useful if not essential. In this paper, 
reference will be made to several types of model. We will explain briefly the most important types that have been produced.

The construction of architectural models aims to predict design aspects of the system based on functional demands and physical principles. In these models, optimization principles are generally applied, but it should not be assumed a priori that the system has actually reached optimality. Natural selection is a mechanism that enables optimization. It is often assumed that an improved performance of an organ contributes positively to the expected number of successfully reproducing offspring. However, animals are complex integrated systems with many functional components. Conflicts and compromises between the optimality of subsystems are therefore expected. The variety of architectural solutions found in protrusible muscular systems can only be understood fully if all physical, phylogenetic and ontogenetic factors are considered. An example of an architectural model that will be discussed below is the model of the accelerator muscle in the chameleon tongue (VAN LEEUWEN, 1997).

In inverse dynamics models, forces and stresses are estimated from measured positions, velocities and accelerations of a collection of material points. Inverse dynamics models include often the following steps:

1. Record positions of landmarks on the body (from film or video analysis).

2. Obtain velocities and accelerations by differentiation.

3. Obtain forces and moments from Newton's Second Law, which relates force to mass and acceleration $(F=m a)$.

The problems that generally occur are the large errors in the calculated values of accelerations due to unavoidable errors in the recorded position s and the indeterminate nature of the force distributions (several force distribution s may often satisfy the recorded movements).

In forward dynamics, the deformation of the system is calculated given some input to the system. Motions of body parts are calculated from the forces generated by the muscles, or the neural control signals. This approach follows more closely the information flow in the body than does the inverse method. However, in practice many difficulties arise due to the complexity of the model and many unknown or poorly-defined parameters.

So far, few investigators have attempted to model the mechanics of muscular protrusible systems with forward dynamics. A relatively simple model was proposed for the tongue of lizards by CHIEL et al. (1992) in which circumferential and longitudinal muscle units were distinguished. The tentacles in squid were modelled with a distributed lumped mass approach by VAN LEEUWEN \& KIER (1997). The human tongue has been 
elegantly modelled by a continuums approach in combination with the finite element method by WILHELMS-TRICARICO (1995). This complex three-dimensional model was designed to study the role of the tongue in speech. The very high computational load of this model limits the number of elements that can be used and therefore the accuracy by which muscle fibre arrangements can be described. Recently, a finite element model for muscle tissue was proposed by JOHANSSON \& BLICKHAN (2000) that yields essentially the same results for the extension of the tentacle as that of VAN LEEUWEN \& KIER (1997). Architectural optimization studies with this model are still unpractical due to the very high computational load. The complexity of a model should be adjusted to the questions to be solved. The dynamic models must necessarily include several architectural features and thus overlap with the architectural models mentioned above. Predictions of optimized architectures often depend heavily on surveys of the consequences of architectural changes for the dynamic performance (see for example VAN LEEUWEN \& KIER, 1997).

\section{COMPARATIVE BIOMECHANICS OF MUSCULAR PROTRUSIBLE ORGANS}

\section{Tentacles in squid}

Squid have two extensible tentacles, which are used in prey capture, and eight arms. The gross-anatomy and histology of the arms and tentacles in squid have been described by KIER (1982). A summary of the tentacular morphology is shown in figure 1 . The tentacles have a proximal muscular stalk that can elongate up to $80 \%$ and a terminal club that is used to adhere to the prey with arrays of suckers. Transverse contractions have the greatest effect on elongation of the stalk. The extensor muscle fibres are indeed arranged in transverse directions and also include a layer of circumferential muscle fibres. Extensor fibre shortening must result in elongation of the stalk due to the local conservation of muscle volume. Longitudinal muscle fibres shorten the tentacles or decelerate an extension, and helical fibre groups rotate the club about its longitudinal axis, and contribute also to other movements. KIER (1982) described qualitative models for the function of the various muscle fibre groups.

To capture prey, a squid swims forward, flares six of the arms outward and backward, and elongates the tentacles within 20-50 ms. Two arms are initially aligned along the tentacles perhaps to provide support as the tentacles extend (KIER \& VAN LEEUWEN, 1997). VAN LEEUWEN \& KIER (1997) proposed a forward dynamics model of the tentacle strike in squid that included mass inertia and a longitudinal array of disc-shaped 
(a)

(b)
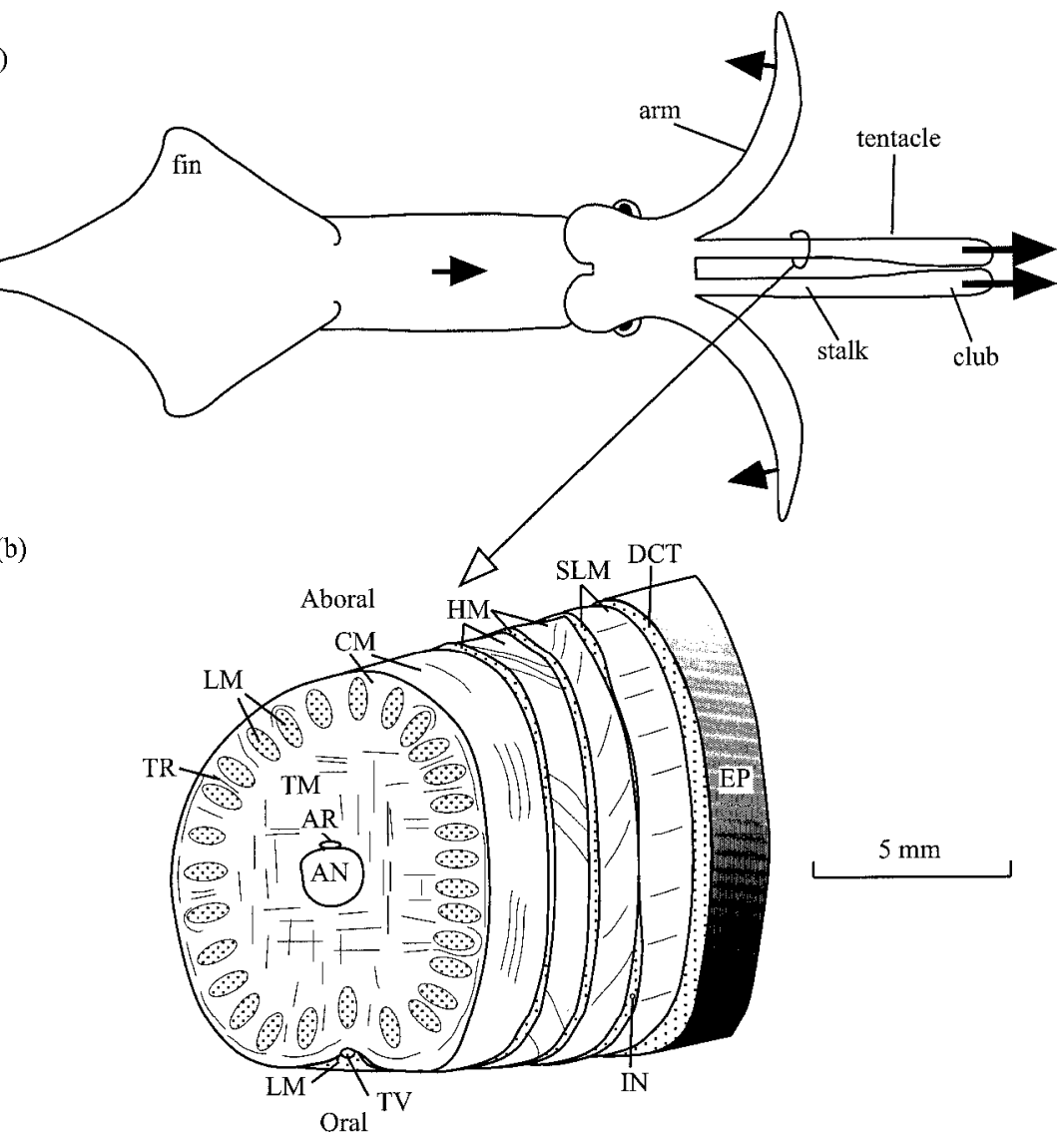

Fig. 1. (a) Diagram of a squid with two of the eight arms shown. During prey capture, the squid swims forward while it flares six arms backwards to gain forward momentum. Two arms (not shown) are used to stabilize the rapidly extending stalks of the two tentacles. (b) Schematic drawing of the morphology of the tentacular stalk in a loliginid squid (redrawn from KIER (1982)). Abbreviations: AN: axial nerve cord; AR: artery; CM: circular muscles; DCT: dermal connective tissue; EP: epithelium; HM: helical muscle; IN: intramuscular nerve cord; LM: longitudinal muscle; SLM: superficial longitudinal muscle; TR: trabeculae of transverse muscle; TM: Transverse muscle; TV: superficial tentacular vein. Complete figure based on figure 1 of VAN LEEUWEN \& KIER (1997).

segments (maximum number used was 51), each receiving an activation signal as input. The terminal club was defined as the most distal element. The geometry of the model is shown in figure $2 b, c, d$. The masses were assumed to be concentrated at the segment boundaries (fig. 2c, d). At the outer boundary, a small mass was added to include the effect of the 
(a)

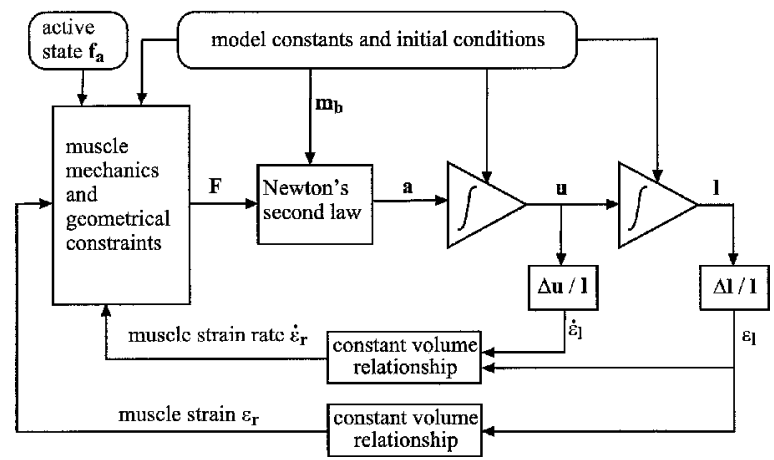

(b)
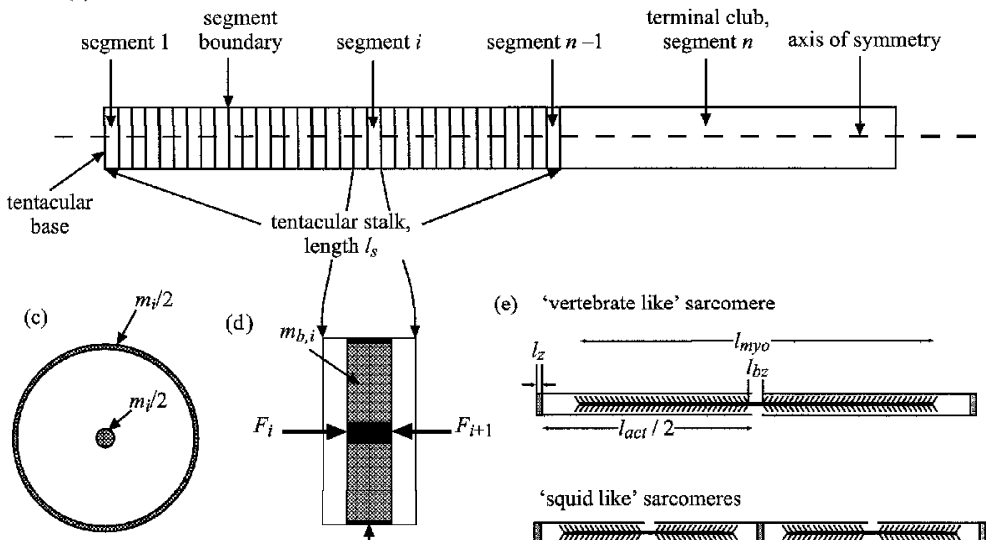

(d)

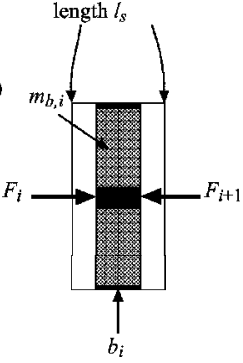

(e) 'vertebrate like' sarcomere

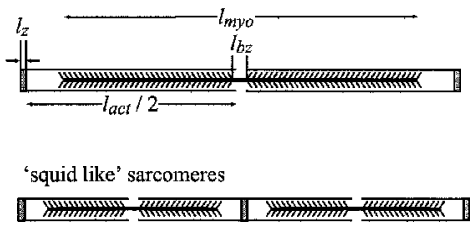

Fig. 2. (a) Simplified flow diagram of the forward dynamics approach applied to the tentacular extension problem by VAN LEEUWEN \& KIER (1997). The tentacle accelerates forwards due to a negative pressure gradient in the distal direction. (b) Geometry of the model, with a tentacular stalk divided in $n-1$ discs and a distal tentacular club. (c) Cross-section of segment with half of the segmental mass assumed to be concentrated in the centre while the other half is concentrated as a ring at the segmental boundary. (d) Sagittal view of the two neighbouring segments with the masses and longitudinal forces indicated. (e) Myofilament lengths in vertebrate like sarcomeres compared to those of the sarcomeres in the tentacular extensor muscles of the squid. Panels (a)-(e) are based on figure 3a, figure 2a, c, d and figure 4a of VAN LEEUWEN \& KIER (1997). Symbols: $\mathbf{f}_{\mathbf{a}}$ : active state vector of muscle segments; $\mathbf{F}$ : force vector on lumped masses (fibre forces and pressure forces); $\mathbf{m}_{\mathbf{b}}$ : boundary masses between segments; $\mathbf{a}$ : acceleration vector of the lumped masses; v: velocity vector of the lumped masses; l: length vector of the segments; $\varepsilon_{l}$ : longitudinal strain of the segments; $\varepsilon_{l}$ : longitudinal strain rate of the segments $\varepsilon_{r}$ : longitudinal strain of the muscle fibres; $\varepsilon_{r}$ : longitudinal strain rate of the muscle fibres; $l_{a c t}$ : length of actin filament length; $l_{b z}$ : length of bare zone on myosin filament; $l_{m y o}$ : length of myosin filament length; $l_{z}$ : width of z-disc. 
surrounding water. The added mass was chosen to be only $8 \%$ of the tentacular mass because of the slender shape and the short duration of the extension. A linear forward motion of a tentacle results in the lowest drag and is therefore the only feasible option in water. It is vital for the animal to maintain the elongate shape as long as possible before the prey is hit, i.e. to avoid substantial bending and the resulting greatly increased drag. A flipping motion of the tentacle during prey capture (as used by inertial tongue elongation in anurans, fig. 5) is not an viable option in the aquatic environment.

Figure 2a shows a simplified flow diagram of the calculation s made by VAN LEEUWEN \& KIER (1997). In short, the active state of the muscle fibres $\left(\mathbf{f}_{\mathbf{a}}\right)$ was prescribed. The forces on the masses $(\mathbf{F})$ result from tensile forces of muscle fibres and connective tissue and intramuscular pressure differences between neighbouring segments. With known values for the lumped masses $\left(\mathbf{m}_{\mathbf{b}}\right)$, the acceleration of the masses (a) can be calculated from Newton's Second Law. Velocities (u) and locations (l) of the masses are obtained by subsequent integrations. From velocities and locations, strains and strain rates can be calculated that are fed back in to muscle mechanics equations to calculate muscle forces.

The model simulated the extension velocity and acceleration of the tentacle accurately as is apparent from the comparison between experiment and simulation of figure 3. Several parameters were estimated because actual values for them were not available and others were adjusted within a normal physiological range. A peak acceleration of approximately $250 \mathrm{~m} . \mathrm{s}^{-2}$ was reached, which overlaps with the accelerations recorded for the chameleon tongue.

During the strike, the muscular stalk of the tentacle accelerates forward only a relatively low 'external' mass (the terminal club plus the small added mass of water). As mentioned above, the extensor muscle fibres are arranged optimally in transverse planes. Variation of the kinematic and force transmission at the level of the muscle fibre arrangement is therefore negligible. The low macroscopic load results therefore also in a relatively low load on the sarcomeres. VAN LEEUWEN \& KIER (1997) calculated that relatively short sarcomeres (as actually found in the extensor fibres, KIER 1985, 1991, 1996) result in the highest extension speeds. The short filament lengths guarantee an average cross-bridge load that results in a favourable power output. Muscle fibres with short sarcomeres are less forceful than those with long sarcomeres but have a greater number of sarcomeres arranged in series per unit length. Since the shortening velocity of elements in series is additive, fibres with short sarcomeres are capable of higher strain rates at small loads (fig. 2e). These statements are illustrated in figure $4 \mathrm{a}$ that shows a contour plot of the peak tentacle 

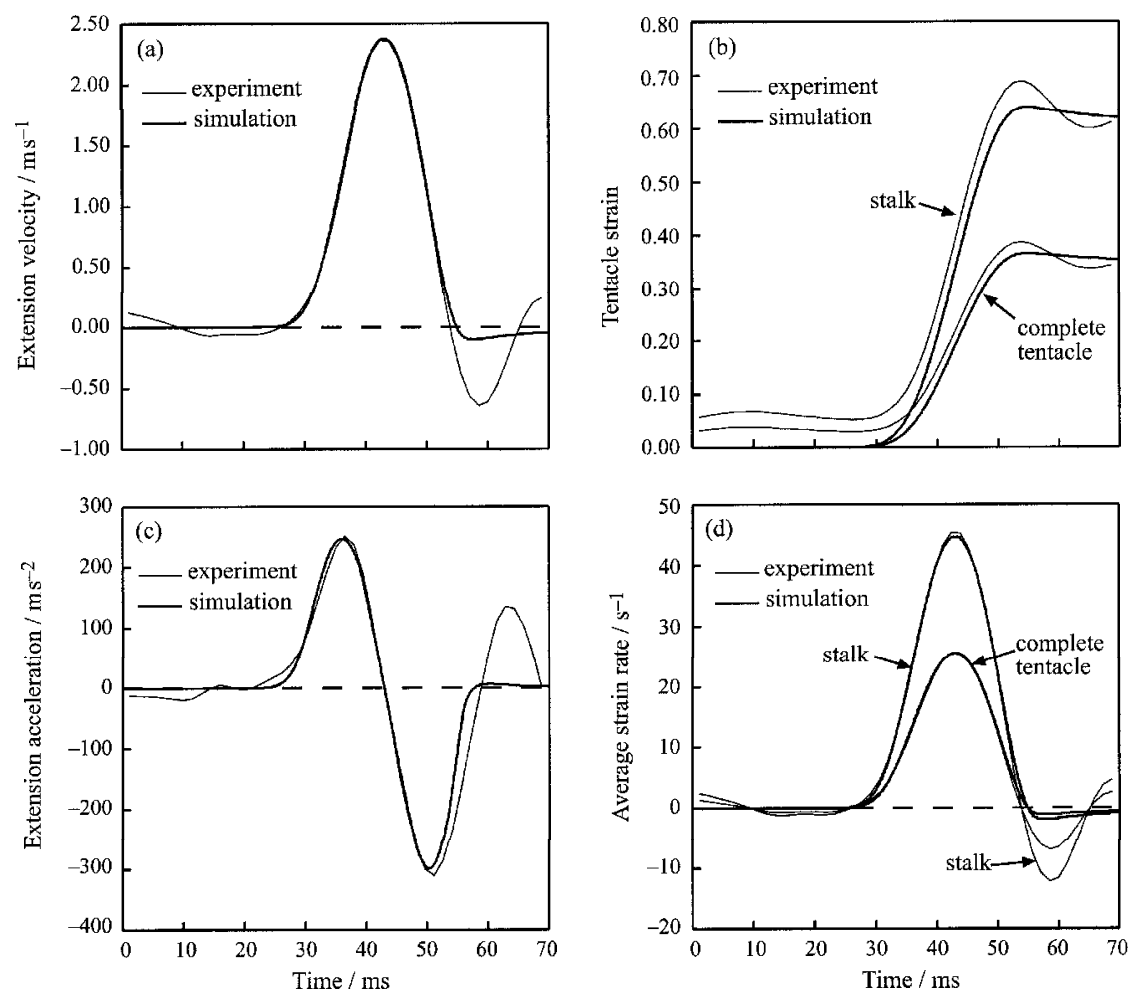

Fig. 3. Comparison between experimental observation and simulation results of tentacle extension in Loligo pealei. (a) Velocity of tentacular tip with respect to the base of the tentacle. (b) Average longitudinal strain of the complete tentacle and the tentacular stalk, respectively. (c) Acceleration of tentacular tip with respect to the base of the tentacle. (d) Average longitudinal strain rate of the complete tentacle and the tentacular stalk, respectively. Panels (a)-(d) are based on figure 7c-f of VAN LEEUWEN \& KIER (1997).

extension speed as a function of the myosin filament lengths at the tentacle base and tip (assuming the length varies linearly between these two sites). The black star in figure $4 \mathrm{a}$ indicates the global maximum of $2.38 \mathrm{~m} . \mathrm{s}^{-1}$, with a myosin length of $0.97 \mu \mathrm{m}$ at the base and $0.50 \mu \mathrm{m}$ at the tip. The black cross indicates the same mean myosin filament length $(0.74 \mu \mathrm{m})$ but now for a constant value along the stalk. The peak extension speed with a constant filament length is still approximately $99 \%$ of the global optimum. The performance would, however, be significantly decreased to $85 \%$ of the global maximum if the myosin filaments were the same length (approximately $1.58 \mu \mathrm{m}$ ) as those found in vertebrate muscle fibres (open star top right corner). Figure $4 \mathrm{~b}$ shows a similar contour plot for the peak kinetic energy of the terminal club. In this analysis it was assumed that (1) 
(a)

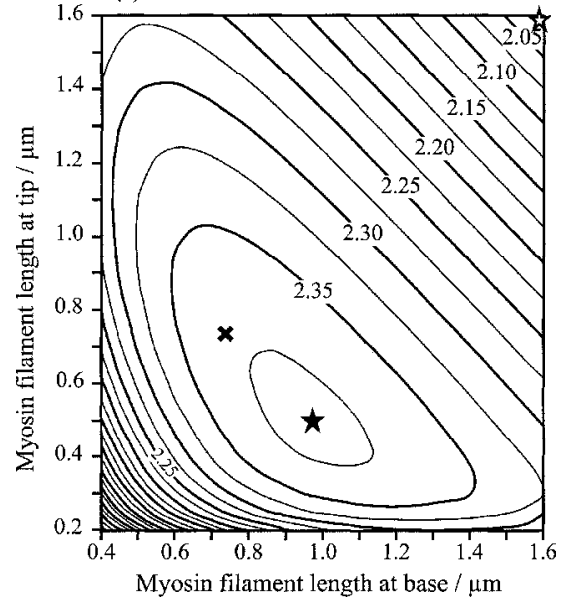

(b)

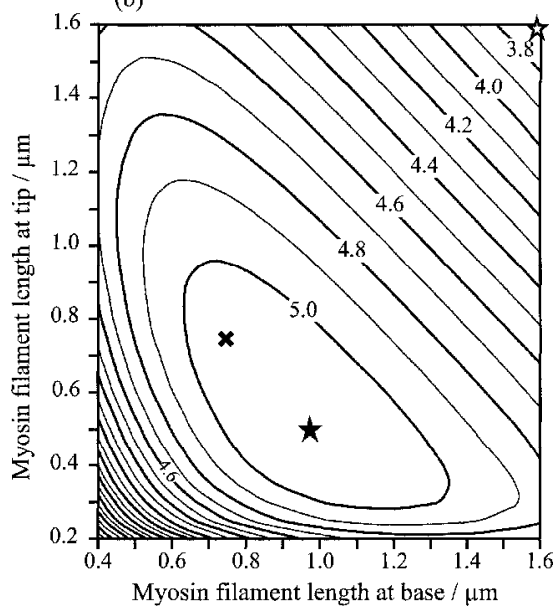

Fig. 4. (a) Contour plot of the calculated peak velocity (in $\mathrm{m} \cdot \mathrm{s}^{-1}$ ) of the terminal club of a squid tentacle, showing the effect of the proximal and distal thick filament lengths. (b) Idem, but now the contours show values of the peak kinetic energy of terminal club in mJ. Filled star: location of global optimum; Open star: location of vertebrate sarcomere design. Cross: constant sarcomere length over tentacular stalk, taking the average of the

optimized case (filled star). Based on figure 10 of VAN LEEUWEN \& KIER (1997).

the stalk had a constant cross-sectional area along its length, and (2) the extensor muscles occupied the same fraction of the cross-section along the length of the tentacle. VAN LEEUWEN \& KIER (1998) showed (their figure 1) that the extensor muscle area tends to decrease slightly (up to 10 to $15 \%$ ) along the stalk in Sepiotheutis lessoniana. If this is also true of Loligo, then it implies that the optimal filament lengths would be slightly underestimated by the model, especially for the distal locations.

To simulate the tentacular motion accurately, VAN LEEUWEN \& KIER (1997) used physiological properties of the muscle fibres that were close to the limits of the physiological range (both the activation time and the maximum tensile stress). In the absence of the required data, it cannot be excluded that the fibre performance was overestimated. If this is the case, a quick release mechanism may be necessary to produce the extraordinary perfomance observed. A pre-stress in the extensor fibres could possibly be generated before the actual extension by coactivation of the longitudinal muscles. This would result in an improved initial acceleration and a higher peak extension velocity. Such a mechanism would be even more effective if elastic energy could be stored in connective tissue in the tentacle prior to its release. Such a mechanism would, however, require unusually rapid deactivation of the longitudinal muscles. 
The following conclusions can be drawn for the tentacle extension in squid:

1. For squid, a flipping movement of the tentacle is not a viable option due to the aquatic environment.

2. In squid, the extensor muscles have an optimal orientation in transverse planes. Kinematic and force transmission are fixed at the gross architectural level.

3. In squid, the myofilament lengths are adjusted to a low load and a high extension speed. The performance is fairly insensitive to filament length changes in the neighbourhood of the configuration for maximum performance.

4. An elastic mechanism is not required if the muscle fibres have extreme physiological properties. Measurements of the physiological properties of the muscle fibres are needed. An elastic mechanism would be required if the muscle fibres are found to have more 'average' properties. Further research is needed to explore this interesting issue.

\section{Tongue protrusion in frogs}

The protrusible tongues of frogs have received considerable attention over the last 20 years. The neuromuscular control of tongue protrusion, including several biomechanical aspects, was recently reviewed by N ISHIKAWA (1999b). Similar to the tentacles of squid, frogs lack an internal tongue skeleton, although the muscular arrangement of the tongue is very different. The tongue is attached anteriorly to the mandibular symphysis. Two pairs of extrinsic muscles are present in all frogs (fig. 5a; HORTON, 1982). The $\mathrm{m}$. genioglossus, the tongue protractor, has its origin on the mandible, adjacent to the mandibular symphysis and inserts in the tongue pad. The $\mathrm{m}$. hyoglossus, the retractor, has its origin on the posteromedial process of the hyoid and inserts along the lateral margin of the tongue pad. In both muscles, the muscle fibres are mainly oriented longitudinally. The biomechanics of tongue protrusion appears to be quite variable among frogs. NisHIKAWA $(1997,1999 a, 1999 b)$ has distinguishe d three protraction mechanisms, mechanical pulling, inertial elongation and hydrostatic elongation.

In mechanical pulling, the tongue actually shortens during protraction due to contraction of the superficial $\mathrm{m}$. genioglossus. The tongue is pulled upwards and forwards and the tongue extends only a few millimetres beyond the lower jaw symphysis at maximum protrusion. The frogs that use this technique generally lunge forward with their body to capture the 
(a)

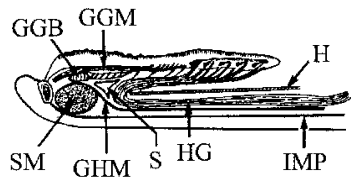

(b)
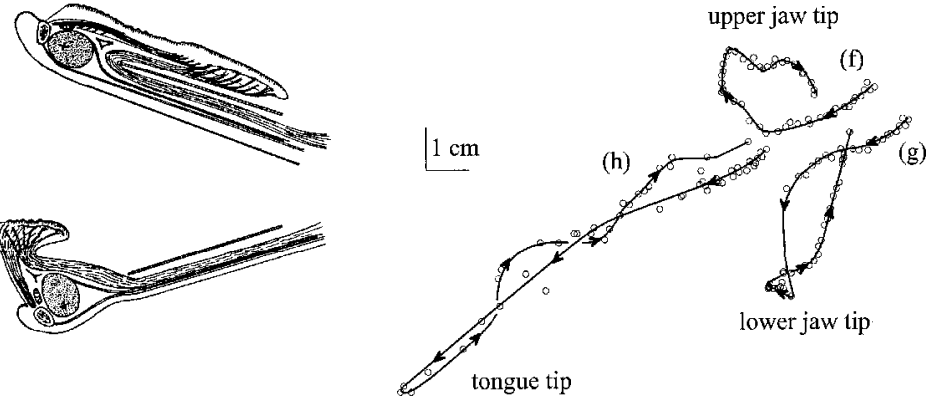

(d)

(c)
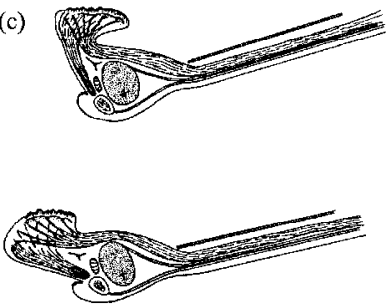

tongue tip

\section{(e)}

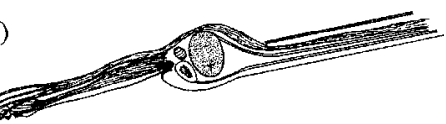

Fig. 5. Inertial tongue elongation in the toad Bufo marinus. (a)-(e) Sagittal sections through the tongue and lower jaw. (a) Tongue at rest. (b) Elevation of the lower jaw (with the skull) and tongue prior to protrusion of the tongue. (c) Lower jaw is rotated downwards and the $\mathrm{m}$. genioglossus contracts and as a result the tongue flips over the mandible (c-e). (f) Movement trajectory of the upper jaw tip. (g) Idem, but for the lower jaw tip. (h) Idem, but for the lingual tip. Note that the lingual tip follows a path close to a straight line. Abbreviations. GGB: m. genioglossus basalis; GGM: m. genioglossus medialis; GHM: geniohyoideu s medialis; SM: m. submentalis; S: lingual sinus; H: hyoid; IMP: m. intermandibularis posterior. Further explanations in the main text. Panels (a)(e) and (f)-(h) are based on figure 1 from GANS \& GORNIAC, 1982 and figure 6 of NisHIKAWA, 1999, respectively.

prey. The tongue reaches only moderate velocities (less than $0.5 \mathrm{~m} . \mathrm{s}^{-1}$ ) and accelerations (less than $10 \mathrm{~m} . \mathrm{s}^{-2}$ ), measured relative to the lower jaw.

In inertial elongation (fig. 5), the tongue shortens initially ( similar to the mechanical pulling mechanism) and rotates subsequently over the lower jaw symphysis, while it elongates to a longitudinal strain of $80 \%$ due to inertial effects, which is similar to the maximum found in tentacles. The motions of the lower jaw play a crucial role in increasing the momentum of the tongue, and hence its extension. An example of this mechanism is found in Bufo marinus (fig. 5). First, the head is elevated (fig. 5a, b), 
then the mouth is opened by a rapid downward rotation of the lower jaw (fig. 5a-e) that conveys momentum to the tongue and results in a ballistic motion. Quite remarkably, the tongue tip follows an almost straight line during protrusion (fig. 5h). Significant energy from muscular systems outside of the tongue is used to generated tongue tip velocities up to $4 \mathrm{~m} . \mathrm{s}^{-1}$ in some frogs with peak accelerations of approximately 300 $\mathrm{m} . \mathrm{s}^{-2}$. From the starting position onwards (fig. 5b), the tongue shortens while it rotates forward over the lower jaw. The shortening of the tongue probably reduces the moment of inertia and could therefore facilitate the flipping motion. A quantitative model is needed to explore this aspect further.

The third mechanism has been denoted as muscular hydrostatic elongation of the tongue. The term 'hydrostatic' is not entirely appropriate because of the dynamic nature of the deformation. Similar to the tentacle in squid, active muscle fibres in the extensor muscle produce a pressure gradient perpendicular to the fibre direction that drives the tongue forward. The morphology and kinematics of this mechanism has been studied in greatest detail in the pig-nosed frog Hemisus marmoratum (NISHIKAWA, 1999b; NishiKAWA et al., 1999). The m. genioglossus consists of two compartments in this frog: (1) a pars longitudinali s (GGL) with two layers of longitudinal muscle fibres that extend between the dentary bone and the tongue tip and (2) a pars dorsoventralis (GGD) with dorsoventrally oriented muscle fibres that extend between the two layers of the pars longitudinalis. The GGD is enveloped by a connective tissue sheet that can be elongated easily. The sheet prevents, however, significant widening of the muscle. Similar to the two other tongue protraction types, the GGL causes an initial tongue protraction in which the tongue is rotated over the mandible. The tongue then elongates probably by subsequent contraction of the GGD. A decrease of the dorsoventral width of the muscle must result in elongation due the connective tissue sheet that constrains lateral extension and the constancy of muscle volume. In contrast to the combined circumferential and bidirectional muscle fibre arrangement in the tentacles in squid, the GGD is locally limited to a single fibre direction. This leads to a much smaller longitudinal strain for a given (negative) strain in the extensor muscle compared with the tentacle. If it is assumed that the lateral width of the GGD is constant, then the longitudinal strain $\varepsilon_{l}$ can be calculated from the longitudinal muscle fibre strain $\varepsilon_{f}$ as:

$$
\varepsilon_{l}=-\varepsilon_{f}\left(1+\varepsilon_{f}\right)^{-1}=-\left(\varepsilon_{f}+\varepsilon_{f}^{2}\right) /\left(1+\varepsilon_{f}\right)^{2} .
$$

It is interesting to compare this formula with that derived by VAN LEEUWEN \& KIER (1997) for the squid tentacle:

$$
\varepsilon_{l}=\left(1+\varepsilon_{f}\right)^{-2}-1=-\left(2 \varepsilon_{f}+\varepsilon_{f}^{2}\right) /\left(1+\varepsilon_{f}\right)^{2} .
$$


The lower and upper curves of figure 6b represent formulae (1) and (2) in graphical form. The longitudinal strain rises more rapidly with a more negative muscle fibre strain in the tentacle than in the frog. In the tentacle, $80 \%$ elongation is reached at a muscle fibre strain of approximately -0.26 . In the tongue, this would require a muscle fibre strain of -0.45 , which is beyond the typical physiological range for vertebrate muscle fibres. Extension would in practise be limited to about 60\% (using equation 1 and a minimum muscle strain of -0.37 ). Nevertheless, the reported extension in the frog is approximately $100 \%$. How can this paradox be solved? NisHIKAWA et al. (1999) have suggested that the initially folded tongue could unfold during its extension. In addition, the extension could have been slightly overestimated due to limitations in the video resolution of the recorded movements.

Now we wish to explore a theoretical architectural space that sheds light on the design of the frog. Although perhaps not exploited by the frog, we wish to discuss a mechanism that could result in a significantly increased extension (compared with formula (2)) with only one muscle fibre orientation. Consider a small rectangular block of muscle tissue in the resting configuration of width $w_{0}$, length $l_{0}$, and height $l_{f 0}$ (fig. 6a). Let the muscle fibres be oriented in the vertical direction, their length is assumed to be identical to $l_{f} 0$. We assume the muscle block to be covered at the lower and upper side by a thin tendinous sheet. The thickness of the sheets will be ignored in the following calculations. Let us assume that straight diagonal collagen fibres are present of resting length

$$
l_{c 0}=l_{0} /\left(\sin \alpha_{0}\right)=w_{0} /\left(\cos \alpha_{0}\right),
$$

where $\alpha_{0}$ is $\arctan \left(l_{0} / w_{0}\right)$. Let us now assume that all muscle fibres in the muscle block contract uniformly to dimensions $\left(l_{f}, w, l\right)$, that the volume of the muscle block remains constant, that the initial rectangular shape is conserved, and that the collagen fibres are free to reorient. From the constancy of volume it follows that:

$$
l_{f} l w=l_{f 0} l_{0} w_{0} .
$$

Hence:

$$
\begin{aligned}
l_{f} / l_{f 0} & =l_{0} w_{0} /(l w)=\left(l_{c 0}^{2} \sin \alpha_{0} \cos \alpha_{0}\right) /\left(l_{c}^{2} \sin \alpha \cos \alpha\right) \\
& =\left(l_{c 0}^{2} \sin 2 \alpha_{0}\right) /\left(l_{c}^{2} \sin 2 \alpha\right) .
\end{aligned}
$$

Now, the angle of the collagen fibres in the sheet with the transverse direction can be derived as:

$$
\alpha=\arcsin \left(\sin 2 \alpha_{0} /\left(\left(1+\varepsilon_{f}\right)\left(1+\varepsilon_{c}\right)^{2}\right)\right) / 2
$$




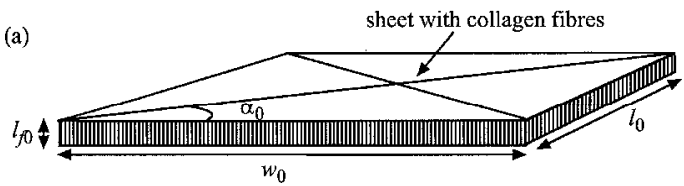

(b)

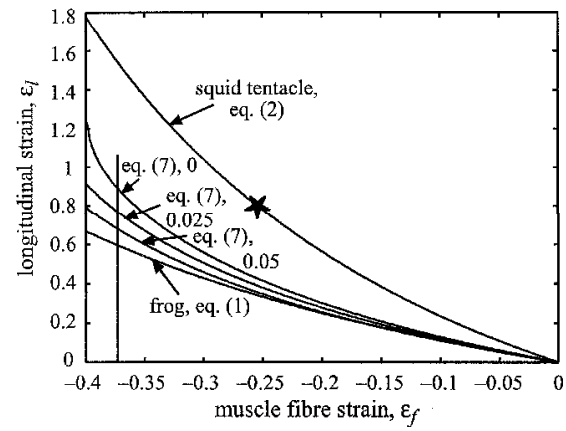

(c)

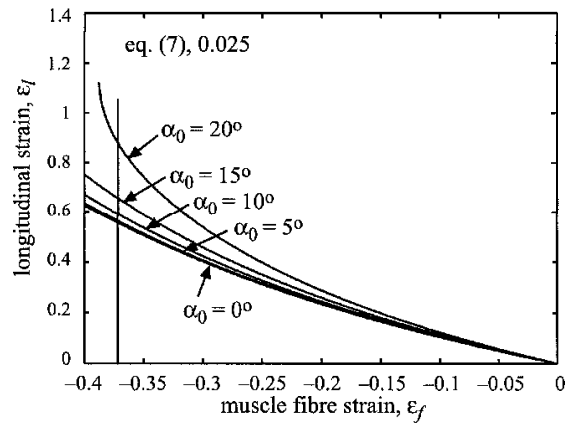

Fig. 6. (a) Muscle block as discussed in the text. (b) Relationships between strain of the extensor muscle fibres and the longitudinal strain of the tentacular stalk (upper curve, eq. 2), and various designs with tendinous sheets and dorsoventrally oriented muscle fibres. The initial collagen fibre angle $\alpha_{0}$ is $18.5^{\circ}$. Abbreviations: eq. (7), 0 : calculation according to equation (7), with inextensible collagen fibres; eq. (7), 0,025 and 0.05: calculation according to equation (7) with maximum collagen fibre strain of 0.025 and 0.05 respectively. Bottom curve shows the longitudinal strain for a constant width of the muscle (eq. 1), as assumed by Nis HiKAWA et al. (1999). The star indicates the maximum elongation found in the tentacular stalk of squid. The vertical line shows the minimum feasible strain for the extensor muscle in the pig-nosed frog. (c) Calculation of longitudinal strain of the tongue in relation to the longitudinal fibre strain in a hypothetical extensor muscle for a number of different values of the initial collagen fibre angle $\alpha_{0}$ (using equation (7), and a maximum collagen fibre strain of 0.025 ). The vertical line shows the minimum feasible strain for the extensor muscle in the pig-nosed frog. Further explanation in the main text.

where muscle fibre strain $\varepsilon_{f}=\left(l_{f}-l_{f 0}\right) / l_{f 0}$ and collagen fibre strain is $\varepsilon_{c}=\left(l_{c}-l_{c 0}\right) / l_{c 0}$. Finally, the longitudinal strain can now be calculated:

$$
\begin{aligned}
\varepsilon_{l} & =\left(l-l_{0}\right) / l_{0}=\left(l_{c} \sin \alpha-l_{c 0} \sin \alpha_{0}\right) /\left(l_{c 0} \sin \alpha_{0}\right) \\
& =\left(\left(1+\varepsilon_{c}\right) \sin \alpha-\sin \alpha_{0}\right) / \sin \alpha_{0} .
\end{aligned}
$$

We do not know what the exact collagen fibre extension would be during tongue extension. Therefore, we evaluated the sensitivity of tongue elongation for the strain in the collagen fibres (fig. 6b), with a value for $\alpha_{0}$ of $18.5^{\circ}$. Maximum collagen strains were used of $0,0.025$, and 0.05 . Inextensible collagen fibres yield the greatest longitudinal extension; the greater the collagen fibre strain, the smaller the longitudinal strain because 
of the increase in tongue width for a particular muscle fibre contraction. Slightly extensible collagen fibres with effective initial orientations have the effect of reducing $w$ during contraction of the GGD, and hence increasing tongue length. For comparison, the longitudinal strains are also shown for the tentacular stalk (equation (2)) and that based on equation (1) which corresponds to the description of NisHIKAWA et al. (1999). Figure $6 \mathrm{c}$ shows the effect of the initial angle $\alpha_{0}$ on the longitudinal strain. Decreasing $\alpha_{0}$ from $20^{\circ}$ onwards results in decreasing longitudinal strains. The angle $\alpha$ at maximum tongue elongation has an upper useful limit of $45^{\circ}$. Therefore, values greater than $20^{\circ}$ for $\alpha_{0}$ do not result in a greater maximum elongation. In conclusion, our calculations suggest that a much greater elongation (but less than that of the tentacle) could be obtained if suitable values of $\alpha_{0}\left(15-20^{\circ}\right)$ of the collagen fibres in the circumferential sheet with the longitudinal axis were present. The mechanism requires considerable shear to occur in the sheet. This requirement may have prevented the development of this mechanism in the pig-nosed frog that has $\alpha_{0} \sim 0^{\circ}$.

The maximum extension speed of the tongue of the pig-nosed frog is much less than that of the tentacles of squid. This can be explained by two factors. First, a smaller extension for a given shortening of the extensor fibres is generated than observed in the tentacle. Second, the myosin filaments in the extensor muscle of the frog tongue are similar to those found in other vertebrate skeletal muscle fibres. It could be that the filament lengths are not optimised for a high peak extension velocity although data are lacking thus far. NISHIKAWA (1999b) has argued that the tongue of the pig-nosed frog can be controlled much more precisely than the inertial tongue at the expense of a lower extension speed, as supported by a higher rate of successful strikes and a greater number of motor units in the genioglossu s muscle.

NisHIKAWA (1999b) has also suggested that mechanical pulling is the most primitive type of tongue protraction. The inertial tongue type has evolved several times among different groups of frogs. The 'hydrostatic' tongue has developed once from the inertial type.

The following conclusions can be made for the tongue protraction in frogs:

1. Frogs do not take advantage of a tongue skeleton to generate high extension speeds of the tongue. Three main tongue protraction mechanisms have been distinguishe d (NISHIKAWA, 1999b).

2. The basic 'pulling' tongue has a limited protraction. Forward body motion is important in the prey capture repertoire.

3. The inertial frog tongue makes a flipping motion that is heavily assisted by jaw movements. 
4. The muscular 'hydrostatic' tongue contains extensor muscle fibres oriented dorsoventrally at right angles to the longitudinal direction. The myo-filament lengths do not seem to be optimised for high extension speeds. A lower extension speed has been observed compared to that of tentacles of squid.

5. The most elaborate control is found in the muscular 'hydrostatic' tongue type.

\section{Tongue projection in Hydromantes, Plethodontidae}

The most elaborate tongue protrusion mechanism in salamanders is found in the family Plethodontidae (LOMBARD \& WAKE, 1977). The most extreme performance is found in the genus Hydromantes (DEBAN et al., 1997), with a tongue protrusion of $80 \%$ of body length (lasting less than $10 \mathrm{~ms}$, in Hydromantes supramontis ). Similar to the chameleon, a sticky tongue pad is used to adhere to the prey. A forked hyobranchial skeleton (a derivative of the visceral skeleton) is present in the tongue, consisting of seven interlinked cartilaginous elements (fig. 7a). An unpaired basibranchial cartilage supports the tongue pad. Paired first and second ceratobranchials articulate with the caudal half of the basibranchial. The ceratobranchials articulate with the posterior paired epibranchials which are enveloped by the tongue protractor muscles, the paired $\mathrm{m}$. subarcualis rectus (SAR). The hyobrancial skeleton folds medially during the initial protraction phase and in Hydromantes is projected out of the mouth with the tongue pad (fig. 7b). The SAR is thought to generate the force for the projection (DEBAN et al., 1997). The epibranchial elements taper caudally. The muscle fibres of the SAR are arranged circumferentially around the epibranchials and are proposed to exert forward pulling forces as well as 'squeezing' forces on the epibranchials. The squeezing forces result in a forwardly directed force because of the tapering of the skeleton. So far, detailed kinematic and dynamic analyses of the tongue protrusion are lacking.

DEBAN \& DICKE (1999) recorded the electrical activity of three muscles involved in the tongue projection and retraction in four species of plethodontid salamanders: (1) the SAR, (2) the m. depressor mandibulae posterior (DMP, a mouth opener), and (3) the m. rectus profundus (RCP, the tongue retractor, which extends between the hyobranchial skeleton and the pelvis. For the largest prey distances (approximately $35 \mathrm{~mm}$ ) it is apparent that the SAR reaches its maximum activity well before maximum activity of the DMP in Hydromantes supramontis (difference is approximately $50 \mathrm{~ms}$ ). The timing difference and the duration of the activity of the SAR increase with prey capture distance. This would allow greater stresses to be generated on the epibranchials at the onset 


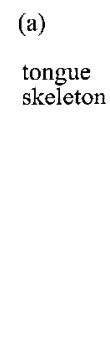

(b) tongue protractor muscles (SAR)

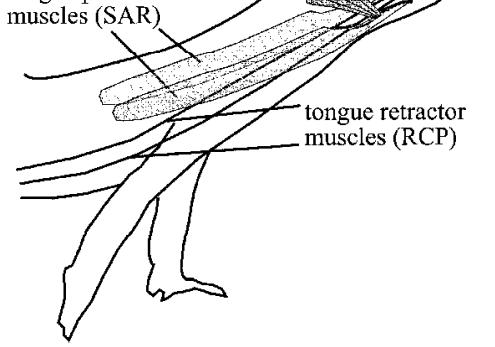

basibranchial

ceratobranchials

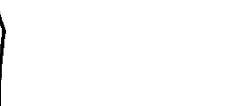

epibranchial

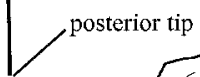

$\gamma$
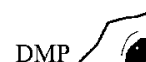

\section{(1)}

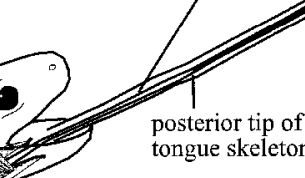

(d)
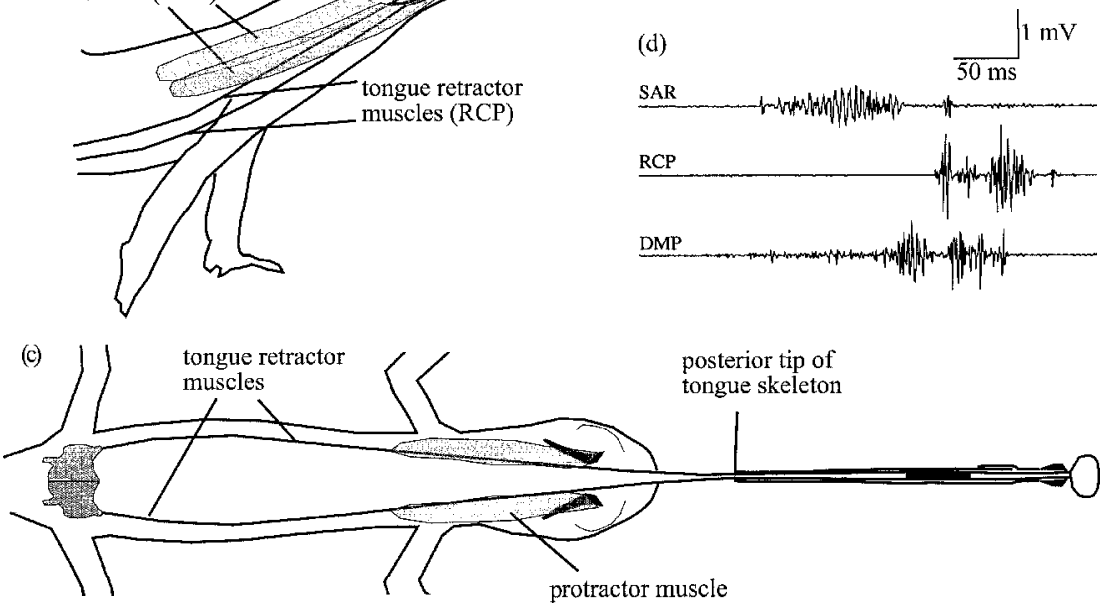

Fig. 7. Tongue projection in Hydromantes supramontis. (a) Dorso-ventral view of the forked hyobranchial tongue skeleton. (b) Schematic drawing of lateral view with fully extended tongue. The tongue skeleton is projected with the tongue pad. (c) Dorsoventral view with extended tongue. Note the extreme length of the tongue retractor that attaches posteriorly to the pelvis. (d) Electromyogram of three muscles involved in tongue projection and release as shown in (b). Initial prey distance was $35 \mathrm{~mm}$. See main text for further explanations. Panels (a)-(c) are redrawn from DEBAN et al. (1997). Panel (d) is based on DEBAn \& DiCKE (1999). Abbreviations. DMP: m. depressor mandibulae; RCP: $\mathrm{m}$. rectus cervicus profundus; SAR: $\mathrm{m}$. subarcualis rectus.

of projection. To our knowledge, an elastic storage mechanism has not been proposed previously for Hydromantes. Nevertheless, the timing characteristics of the electromyograms are in agreement with such a mechanism, in which a spring is loaded by the protractor muscle prior to the projection. A larger prey distance requires more energy to be stored in the spring. This requires earlier initiation and longer duration of activity in the SAR. Both effects are supported by the electromyograms (cf. fig. 3 of DEBAN \& DICKE, 1999). An elastic mechanism as proposed 
here as a possibility does not exclude a contribution of direct propulsive 'squeezing' forces of the SAR.

The following conclusions can be made for the tongue projection in Hydromantes:

1. The paired protractor muscle envelops the tapered posterior elements of the forked hyobranchial tongue skeleton. The tongue skeleton (but not the protractor) is projected out of the mouth with the tongue pad.

2. The performance of the tongue projection is comparable to that of chameleons (see below).

3. It is hypothesized here that an elastic mechanism could be essential for the rapid projection. Electromyographic records are in agreement with this viewpoint. The records agree also with the viewpoint that greater squeezing forces are required for a longer distance. More research is required to settle this issue.

\section{The chameleon tongue}

Chameleons project their tongue during prey capture over a distance of more than one body length. Tongue projection lasts about 20-50 ms, depending on body size and prey distance (BELL, 1990). Qualitative proposals of the projection mechanism have been made, but quantitative mechanical models and experimental tests are needed. The current proposal for tongue projection can be summarized as follows (GANS, 1967; WAINWRIGHT \& BENNETT, 1992a, 1992b). A central elongate entoglossal process is present in the tongue (fig. 8a). This cartilaginous process is enveloped by the cylindrically shaped accelerator muscle, which was identified originally by BRÜCKE (1852) as the main actuator for tongue extension. During tongue projection, the main posterior portion contracts radially, elongates and slides forward along the entoglossal process. Radial contraction must result in elongation because muscle tissue is essentially incompressible. The normal stress produced by the accelerator muscle on the suggested tapering tip of the entoglossal process results in a forwardly directed force component on the muscle that drives the muscle and tongue pad forward until the tongue pad is projected off the entoglossal process. This hypothesis is not yet adequately tested experimentally. Some question s that should be addressed to obtain a better insight in the mechanism are:

1. What is the required power for the movement and how does it compare with the physiological limits of the muscles?

2. Does the shape of the entoglossal process in a wide range of species agree with the force requirements for projection? 


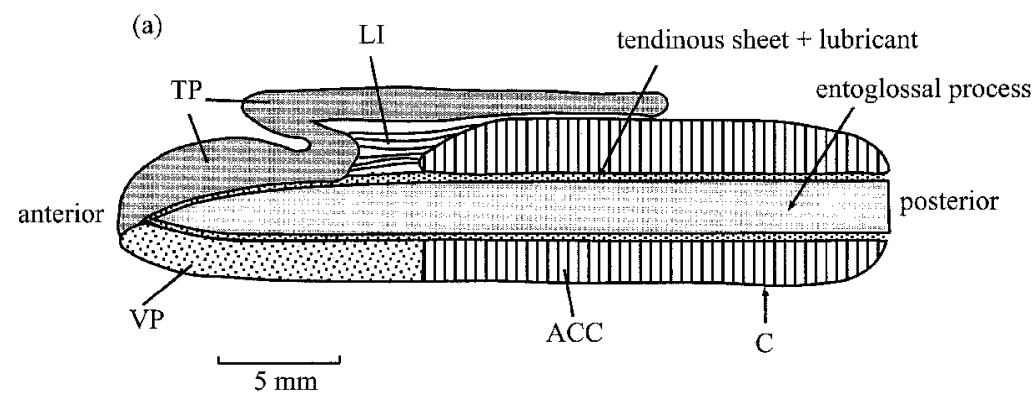

(b)
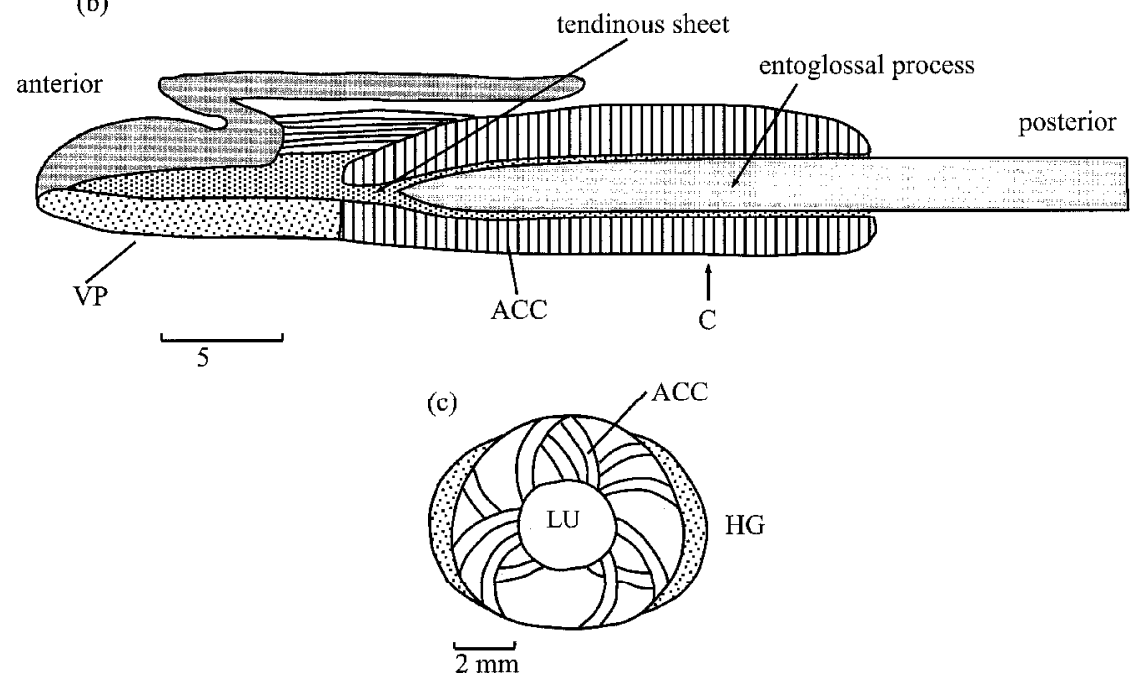

Fig. 8. Schematic drawing of the tongue pad and accelerator muscle of the chameleon tongue. (a) Parasagittal section through the tongue of the chameleon (Chamaeleo jacksonii). (b) Similar to (a), but tongue pad and accelerator muscle has elongated and extends partly beyond the tip of the entoglossal process. (c) Cross-section through the tongue at arrow C in (a). Panels (a)-(c) based on figure 1 of VAN LEEUWEN (1997) and figures from WAINWRIGHT \& BENNET (1992a) and BELL (1989). Abbreviations. ACC: accelerator muscle; HG: hyoglossal muscle; LI: longitudinal adductor muscle; TP: membrana glutinosa of tongue pad; VP: ventral projection of accelerator muscle.

3. During which stage of the process is the accelerator muscle actually activated and when does it elongate?

4. Could an elastic storage mechanism be involved in the tongue motion?

5. Does a forward dynamics model that takes realistic anatomical and physiological data as input accurately predict the observed 
kinematic characteristics such as those reported by WAINWRIGHT et al. (1991)?

There is a striking resemblance between the electromyographic recordings of the paired $\mathrm{m}$. subarcualis rectus (SAR) in Hydromantes supramontis (DEBAN \& DICKE, 1999) and that of the accelerator muscle in Chamaeleo jacksonii (WAINWRIGHT \& BENNETT, 1992a). In both cases, the activation begins $50 \mathrm{~s}$ or more before the actual fast release of the tongue, causing presumably considerable stress in the muscles before tongue projection. The timing of the EMG agrees with the possibility of an elastic energy storage mechanism.

Let us now consider the architecture of the accelerator muscle with muscle fibres arranged in transverse planes. The muscle fibres of the accelerator muscle make spiral-shaped trajectories from the internal collagen sheet to the external collagen sheet (fig. 8c).

Following VAN LEEUWEN (1997), we distinguish a series of functional demands for the accelerator muscle (a subset of his original proposal):

1. The muscle fibres should be arranged according to the principle of close packing, because this provides the highest work and power output per unit mass.

2. Torsional motions that do not contribute to the forward motion should be prevented in the longest projections. At the same time, torsional motion of the tongue pad should be possible if required.

3. A uniform longitudinal muscle fibre strain and a uniform work output per unit mass should be possible throughout the accelerator muscle.

4. The normal stress on the internal collagenous sheet and entoglossal process should be directed inwards.

VAN LEEUWEN (1997) distinguished two theoretical designs (fig. 9), not present in the chameleon, that do not meet all the functional demands mentioned above. Consideration of the disadvantages of these designs provides insight into the necessity of the more complex design found in the chameleon. Figure 9a shows a design in which the muscle fibres are arranged in a set of concentric circular arcs typical of a sphincter. This design provides excellent packing (requirement 1) and allows also for an inwardly directed normal stress on the entoglossal process (requirement 4). A uniform strain distribution in the muscle fibres does not, however, occur during contraction (requirement 3 ). Control of axial rotation by the accelerator muscle of the tongue pad would also be problematic in this design (requirement 2). Figure 9b shows the second theoretical design with radially directed muscle fibres. Here, considerable space is present between the muscle fibres, which is in conflict with requirement 1 and 

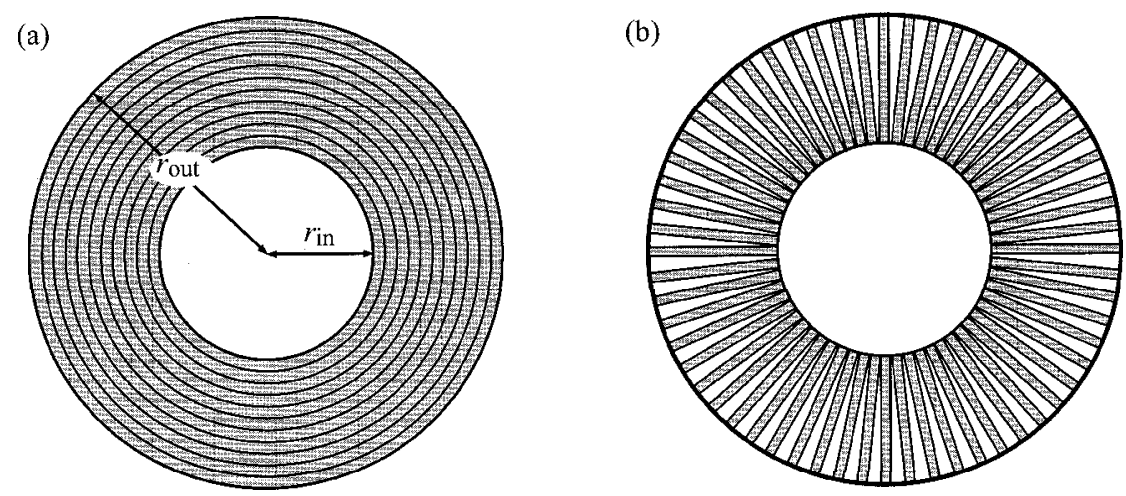

Fig. 9. Illustration of two imperfect theoretical designs of cross-sections of the accelerator muscle that are not found in the chameleon tongue. (a) Design typical for a common sphincter with concentric muscle fibre layers. (b) Design with radially directed muscle fibres. Further explanations in the main text. Based on VAN LEEUWEN (1997).

the normal stress on the entoglossal process is directed outwards (VAN LEEUWEN, 1997).

A design intermediate between these extremes can fulfil all of the requirements (VAN LEEUWEN, 1997). Let us first consider the requirement of close packing of the muscle fibres. Figure 10a shows a diagram for a model fibre that runs from the inner boundary (the outer surface of the inner collagenous sheet) to the outer boundary of the muscle. The muscle is assumed to be delimited by two concentric circular boundaries of radii $r_{i n}$ and $r_{\text {out }}$. Let the muscle fibre have an angle $\alpha_{f, \text { rin }}$ with the radial direction and let it have a constant thickness $d_{f}$ (in the transverse plane) and width (in the longitudinal direction of the tongue) over its length. Thus, the cross-section is constant along the fibre. In the transverse plane, the fibre is attached over the following distance to the inner boundary (VAN LEEUWEN, 1997):

$$
g_{f, \text { rin }}=d_{f} /\left(\cos \alpha_{f, \text { rin }}\right),
$$

with the assumption that $g_{f, \text { rin }} \ll 2 \pi r_{i n}$. The length by which an imaginary circle with radius $r\left(r_{i n} \leqslant r \leqslant r_{\text {out }}\right)$ traverses the muscle is equal to

$$
g_{f}=d_{f} /\left(\cos \alpha_{f}\right) .
$$

A complete filling of the muscle with muscle fibres requires that

$$
g_{f}=g_{f, \text { rin }} r / r_{i n} \text {. }
$$




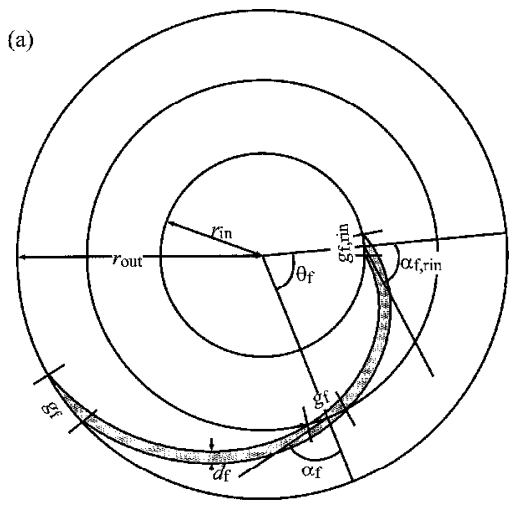

(b) accelerator muscle with parts

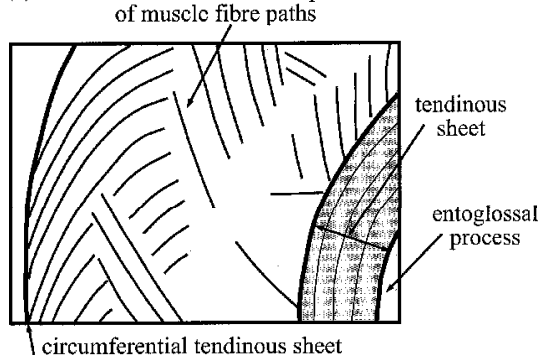

(c)

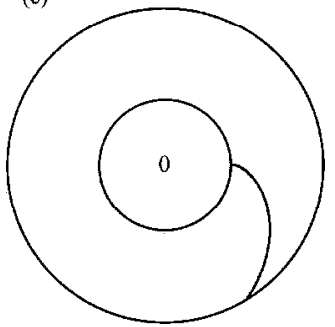

(d)

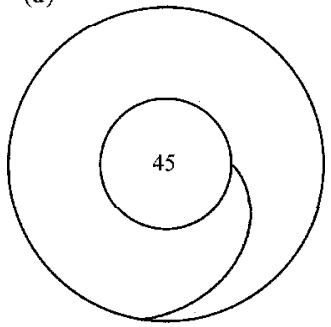

(e)

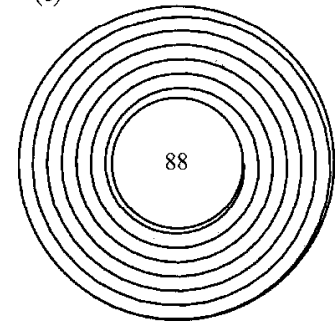

Fig. 10. (a) Diagram for the calculation of fibre trajectory for close packing design according to equation (11). Panels (c)-(e) show three examples of designs that meet the requirement of close packing, with values for $\alpha_{f, \text { rin }}$ of 0,45 and $88^{\circ}$, respectively. Panel (d) shows a design that closely resembles that found in the chameleon as illustrated by panel (b) that shows a drawing based on an approximately transverse histological section through the tongue base by GANS (1967). Further explanations in the main text. Based on VAN LEEUWEN (1997).

From formulae (8), (9), and (10) it can now be derived that

$$
\alpha_{f}=\arccos \left(r_{i n} \cos \alpha_{f, \text { rin }} / r\right) .
$$

Thus, the orientation of the muscle fibre is determined by $r_{i n}$ and $\alpha_{f, \text { rin }}$. Figure 10c-e shows three examples that meet the close packing requirement, with values for $\alpha_{f, \text { rin }}$ of 0,45 , and $88^{\circ}$, respectively. VAN LEEUWEN (1997) calculated that a design with a value for $\alpha_{f, \text { rin }}$ of approximately $45^{\circ}$ results not only in an excellent packing (requirement 1 ), but an almost uniform longitudinal muscle fibre strain can also be realized (requirement 3). Torsional motions can be prevented if equal numbers of fibres run clockwise and anti-clockwise. These predictions are in 
agreement with anatomical observations (VAN LEEUWEN, 1997). Axial rotation of the tongue pad could be produced if one of these fibre groups is more highly activated than the other. Thus, requirement 2 is fulfilled. A small, but inwardly directed, normal stress is expected based on the observed $r_{\text {out }} / r_{\text {in }}$ ratio and inner fibre angles of approximately $45^{\circ}$ (requirement 4; $c f$. fig. 7 of VAN LEEUWEN, 1997). Thus, the arrangement observed in the chameleon appears to meet the requirements outlined above.

We wish to make the following conclusions for the chameleon tongue:

1. In the chameleon tongue, an entoglossal bone is enveloped by the accelerator muscle. The accelerator muscle has been suggested to generated a normal stress on the entoglossal bone. The tongue has been suggested to be projected forward by the reaction force of the entoglossal bone on the muscle and tongue pad. In contrast to the extensor muscle fibres in the squid, very short myofilaments are presumably not required because either (a) the force transmission is essentially different resulting in a higher load of the sarcomeres or (b) the muscle fibres are allowed to contract relatively slowly while loading a spring.

2. The spiral shaped muscle-fibre paths in the accelerator muscle appear to be arranged for close packing and uniform strains (VAN LEEUWEN, 1997).

3. Hypercontraction in the retractor muscle limits its required length.

4. The accelerator muscle extends beyond the tip of the entoglossal in a ballistic motion.

5. The projection mechanism of the chameleon needs to be reconsidered based on criteria of maximum power output of the accelerator muscle, actual shape of the entoglossal bone and role of internal connective tissue sheets.

\section{Retraction}

So far, we have mainly discussed various mechanisms of protrusion of muscular systems that are used during capture prey. Special requirements are needed to successfully contract the organs. The tentacles of squid elongate by about $80 \%$. Relatively long actin filaments in the longitudinal muscle fibre group (fig. 1a) ensure that enough overlap between myosin and actin filaments is present for an effective retraction (KIER, 1996). In addition, the longitudinal muscles consist of obliquely striated fibres, which typically show long range elongation and contraction and a flattened length-tension relationship (HIDAKA et al., 1969; LANZAVECCHIA \& ARCidiaCONO, 1981; Miller, 1975). The longitudinal muscle fibres does not seem to be folded at the initiation of tentacle extension. 
The maximum relative extension found in frogs is similar to that of the tentacles of squid, suggesting that relatively long actin filaments are sufficient as a special architectural requirement. The most extreme tongue projections in the plethodontid salamanders show extensions of several times the length of the protractor muscle. The functional requirement of tongue retraction is fulfilled by a very long retractor muscle that extends between the branchial skeleton and the pelvis (DEBAN et al., 1997). In addition, the retractor muscle is folded before projection occurs. The unusual length of the retractor muscle probably ensures that the strain at full tongue extension remains fairly moderate.

The chameleon has the most extreme tongue projection. Prior to projection, the hyoglossi muscles (the paired retractor muscles: fig. 8c) are folded, which increases the maximum projection distance. In addition, this muscle is known to have a special ultrastructural adaptation to a very large strain range. The thick filaments of the sarcomeres penetrate the perforated Z-discs in a state of hyper-contraction before the tongue is projected. This specialized architectural feature results in the remarkable ratio of maximum extended length over retracted length of approximately 6.2 (RICE, 1973).

\section{GENERAL CONCLUSIONS AND PERSPECTIVES}

In this paper, we have discussed several remarkable muscular protrusible systems that are used to capture prey. Different external media (water and air) and phylogenetic histories have resulted in a remarkable variety of architectures. Currently, we are only beginning to discover the subtleties of these complex neuromuscular control systems. Structural adaptations to the extreme mechanical requirements are found at almost every architectural level. Progress in this field is stimulated best by a combination of detailed morphological and physiological observations, quantitative models of the mechanics and control, and phylogenetic inferences. Relatively little is known about the physiological properties of the muscular systems and the role that elastic energy storage mechanisms may play in projection. Forward dynamics models are not available for most systems, in particular frogs, salamanders and chameleons. Quantitative characterization of the varying strain distribution s in the muscular organs are not available for any system, but are urgently needed.

\section{ACKNOWLEDGEMENTS}

It is a pleasure to dedicate this paper to Professor dr Jan W.M. Osse who played a stimulating role in the scientific career of two of us (JLvL and 
JHdG). Dr Mees Muller is thanked for comments on a draft version of this paper. Dr Kiisa Nishikawa and Dr Steve Deban kindly agreed that material from their previous publication could be used to compose figure 5 ( $c f$. NishiKAWA, 1999b) and figure 7 ( $c f$. DEBAN et al., 1997; DEBAN \& DICKE, 1999).

\section{REFERENCES}

BELL, D.A., 1989. Functional anatomy of the chameleon tongue. Zool. Jb. Anat. 119: 313-336.

BELl, D.A., 1990. Kinematics of prey capture in the chameleon. Zool. Jb. Physiol. 94: 247-260.

BRÜCKE, E., 1852. Über die Zunge der Chamäleonen. Sitz. Ber. Math.-Nat. Kl. Akad. Wiss., Wien 8: 62-70.

Chiel, H.J., P. Crago, J.M. Mansour \& K. Hathi, 1992. Biomechanics of a muscular hydrostat: a model of lapping by a reptilian tongue. Biol. Cybern. 67: 403415.

Deban, S.M., D.B. WaKe \& G. Roth, 1997. Salamander with a ballistic tongue. Nature 389: 27-28.

Deban, S.M. \& U. Dicke, 1999. Motor control of tongue movement during prey capture in plethodontid salamanders. J. Exp. Biol. 202: 3699-3714.

GANS, C., 1967. The chameleon. Nat. Hist. 76: 52-59.

GANS, C. \& G.C. GORNIAK, 1982. Functional morphology of lingual protrusion in marine toads (Bufo marinus). Am. J. Anat. 163: 195-222.

Hidaka, T., H. Kuriyama \& T. Yamamoto, 1969. The mechanical properties of the longitudinal muscle in the earthworm. J. Exp. Biol. 50: 431-443.

HORTON, W.M., 1982. Diversity and systematic significance of anuran tongue musculature. Copeia 1982: 595-602.

Johansson, T. \& R. Blickhan, 2000. A finite element model for the analysis of skeletal muscle. J. Theor. Biol. in press.

KIER, W.M., 1982. The functional morphology of the musculature of squid (Loliginidae) arms and tentacles. J. Morphol. 172: 179-192.

KIER, W.M., 1985. The musculature of squid arms and tentacles: ultrastructural evidence for functional differences. J. Morphol. 185: 223-239.

KIER, W.M., 1991. Squid cross-striated muscle: the evolution of a specialized muscle fibre type. Bull. Mar. Sci. 49: 389-403.

KIER, W.M., 1996. Muscle development in squid: ultrastructural differentiation of a specialized muscle fiber type. J. Morphol. 229: 271-288.

KIER, W.M. \& J.L. VAN LEEUWEN, 1997. A kinematic analysis of tentacle extension in the squid Loligo pealei. J. Exp. Biol. 200: 41-53.

Lanzavecchia, G. \& G. Arcidiacono, 1981. Contraction mechanism of helical muscles: experimental and theoretical analysis. J. Submicrosc. Cytol. 13: 253-266.

LOMBARD, R.E. \& D.B. WAKE, 1977. Tongue evolution in the lungless salamanders, Family Plethodontidae. II Function and evolutionary diversity. J. Morphol. 153: 3980.

Miller, J.B., 1975. The length-tension relationship of the longitudinal muscle of the leech. J. Exp. Biol. 62: 43-53. 
NishiKAWA, K.C., 1997. Emergence of novel functions during brain evolution. Bioscience 47: 341-354.

NishiKaWA, K.C., 1999a. Feeding in frogs. In: K. Schwenk (ed.). Feeding in Tetrapod Vertebrates: Form Function and Evolution. Academic Press.

NishiKaWA, K.C., 1999b. Neuromuscular control of prey capture in frogs. Phil. Trans. R. Soc. B 354: 941-954.

NishiKaWA, K.C., W.M. KieR \& K.K. SMith, 1999. Morphology and mechanics of tongue movement in the African pig-nosed frog Hemisus marmoratum: a muscular hydrostatic model. J. Exp. Biol. 202: 771-780.

RICE, M.J., 1973. Supercontracting striated muscle in a vertebrate. Nature 243: 238-240.

VAN LEeUWEN, J.L., 1997. Why the chameleon has spiral-shaped muscle fibres in its tongue. Phil. Trans. R. Soc. B 352: 573-589.

VAn LeeuWen, J.L. \& W.M. KieR, 1997. Functional design of tentacles in squid: linking sarcomere ultrastructure to gross morphological dynamics. Phil. Trans. R. Soc. B 352: 551-571.

VAn Leeuwen, J.L. \& W.M. KieR, 1998. Dynamics and scaling of the tentacles in squid. Biona Report 13, 9-10.

WAINWRIGHT, P.C. \& A.F. BENNETT, 1992a. The mechanism of tongue projection in chameleons. I. Electromyographic tests of functional hypotheses J. Exp. Biol. 168: $1-21$.

Wainwright, P.C. \& A.F. BennetT, 1992b. The mechanism of tongue projection in chameleons. II. Role of shape change in a muscular hydrostat J. Exp. Biol. 168: 23-40.

Wainwright, P.C., D.M. Kraklau \& A.F. BennetT, 1991. Kinematics of tongue projection in Chamaeleo oustaleti. J. Exp. Biol. 159: 109-133.

WILHELMS-TRICARICO, R., 1995. Physiological modelling of speech production: Methods for modeling soft-tissue articulators. J. Acoust. Soc. Am. 97: 3085-3098. 